\title{
Quantum Oscillations of Elastic Modiuli and Softening of Phonon Modes in Metals
}

\author{
Natalya A. Zimbovskaya ${ }^{a, b}$ \\ ${ }^{a}$ Department of Physics \& Astronomy, St. Cloud State University, \\ 720 Fourth Avenue South, St. Cloud, MN 56301, ${ }^{b}$ Department of Physics and Electronics, \\ University of Puerto Rico at Humacao, CUH Station, Humacao, PR, 00791
}

(Dated: November 20, 2018)

\begin{abstract}
In this paper we present a theoretical analysis of the effect of magnetostriction on quantum oscillations of elastic constants in metals under strong magnetic fields. It is shown that at low temperatures a significant softening of some acoustic modes could occur near peaks of quantum oscillations of the electron density of states (DOS) at the Fermi surface (FS). This effect is caused by the Condon magnetic instability, and it can give rise to a lattice instability. We show that the most favorable conditions for this instability to be revealed occur in metals whose Fermi surfaces include nearly cylindrical segments.

PACS numbers: $73.21 \mathrm{Cd} ; 73.40 .-\mathrm{C}$
\end{abstract}

Experimental data concerning quantum oscillations in various observables in metals under strong magnetic fields were repeatedly used in studies of their electron characteristics. At low temperatures when the parameter $\theta$ is small compared to unity $\left(\theta=2 \pi^{2} T / \hbar \Omega ; T\right.$ is the temperature expressed in units of energy; $\hbar \Omega$ is the value of a cyclotron quantum) these oscillations can exhibit a rich structure. The latter could be analyzed even within a simple isotropic model. For instance, it is known that the longitudinal magnetic susceptibility of a metal can reveal divergencies at peaks of quantum oscillations at low temperatures [1-3]. These divergencies crucially depend on interaction among conduction electrons, and they indicate a possibility of a diamagnetic phase transition in a metal, producing Condon domain structure near the oscillation peaks [4]. Also, it was proposed in some earlier works that the above features in the magnetic susceptibility could give rise to a sensible reduction of elastic moduli in metals $[1,4-6]$. Here, we demonstrate that the effect of softening of phonon modes near the peaks of magnetic quantum oscillations of the electron DOS could be significantly strengthened, and even the relevant structural phase transitions could occur and be observed under feasible experimental conditions in those metals whose Fermi surfaces insert nearly cylindrical belts.

At first, we derive expressions for electron contributions to the elastic constants. To simplify further calculations we assume that the FS is axially symmetric, and the external magnetic field $\mathbf{B}$ is directed along the symmetry axis ("z" axis of the chosen coordinate system). We analyze the elastic response of a metal to an external deformation described with the lattice displacement vector $\mathbf{u}(\mathbf{r})$. The effect of conduction electrons on the crystalline lattice arises due to a self-consistent electric field which appears under deformation. Also, the lattice deformation gives rise to an additional inhomogeneous magnetic field $\mathbf{b}(\mathbf{r})$, and to deformation induced corrections to the crystalline fields. Here, we omit the latter at first steps of our analysis to include them later. The emergence of the electric and magnetic fields accompanying the lattice deformation, leads to a redistribution of the electron density $N$. The local change in the electronic density $\delta N(\mathbf{r})$ equals:

$$
\begin{aligned}
\delta N(\mathbf{r}) & =-\frac{\partial N}{\partial \zeta} e \varphi(\mathbf{r})+\frac{\partial N}{\partial B} \mathbf{b}(\mathbf{r}) \\
& \equiv-N_{\zeta}^{*}\left(e \varphi(\mathbf{r})+\frac{\partial \zeta}{\partial B} \mathbf{b}(\mathbf{r})\right)
\end{aligned}
$$

where $e$ is the absolute value of the electron charge.

The magnetic field $\mathbf{b}(\mathbf{r})$ satisfies the equation:

$$
\begin{aligned}
& \operatorname{curl} \mathbf{b}(\mathbf{r})=4 \pi \operatorname{curl} \delta \mathbf{M}(\mathbf{r}) \\
= & 4 \pi \operatorname{curl}\left(\frac{\partial \mathbf{M}}{\partial \zeta} e \varphi(\mathbf{r})+\frac{\partial \mathbf{M}}{\partial \mathbf{B}} \mathbf{b}(\mathbf{r})\right) \operatorname{div} \mathbf{b}(\mathbf{r})=0 .
\end{aligned}
$$

Here $\mathbf{M}$ is the magnetization vector; $\zeta$ is the chemical potential of charge carriers; $\varphi(\mathbf{r})$ is the potential of the electrical field, arising due to the deformation. The quantity $N_{\zeta}^{*}$ included into Eq.(1) is closely related to the electron density of states (DOS) on the Fermi surface $N_{\zeta}$. The diffference between the two originates from the correlations in the electron system. Within the framework of the phenomenological Fermi-liquid theory the renormalized DOS $N_{\zeta}^{*}$ has the form (See [1]):

$$
N_{\zeta}^{*}=-\left.\sum_{\nu \nu^{\prime}} \frac{f_{\nu}-f_{\nu^{\prime}}}{E_{\nu}-E_{\nu^{\prime}}} n_{\nu \nu^{\prime}}^{*}(-\mathbf{q}) n_{\nu^{\prime} \nu}(\mathbf{q})\right|_{q \rightarrow 0}
$$

where $f_{\nu}$ is the Fermi distribution function for quasiparticles with energies $E_{\nu}$, and $n_{\nu^{\prime} \nu}(\mathbf{q})$ is the Fourier transform of the operator of electron density in space variables. The renormalized operator of electron density $n_{\nu \nu^{\prime}}^{*}(-\mathbf{q})$ is related to the "bare" operator $n_{\nu \nu^{\prime}}(-\mathbf{q})$ as follows:

$$
n_{\nu \nu^{\prime}}^{*}(-\mathbf{q})=n_{\nu \nu^{\prime}}(-\mathbf{q})+\sum_{\nu_{1} \nu_{2}} \frac{f_{\nu_{1}}-f_{\nu_{2}}}{E_{\nu_{1}}-E_{\nu_{2}}} F_{\nu \nu^{\prime}}^{\nu_{1} \nu_{2}} n_{\nu_{1} \nu_{2}}^{*}(-\mathbf{q})
$$


where $F_{\nu \nu^{\prime}}^{\nu_{1} \nu_{2}}$ are the matrix elements of the Fermi-liquid kernel:

$$
F_{\nu \nu^{\prime}}^{\nu_{1} \nu_{2}}=\varphi_{\alpha \alpha^{\prime}}^{\alpha_{1} \alpha_{2}} \delta_{\sigma \sigma^{\prime}} \delta_{\sigma_{1} \sigma_{2}}+\psi_{\alpha \alpha^{\prime}}^{\alpha_{1} \alpha_{2}}\left(\mathbf{s}_{\sigma \sigma^{\prime}} \mathbf{s}_{\sigma_{1} \sigma_{2}}\right)
$$

Here, $\alpha$ is the set of orbital quantum numbers, $\sigma$ is the spin number, and $\mathbf{s}$ is the operator of the electron spin.

The relations (1), (2) have to be complemented by the condition of electrical neutrality of the system :

$$
\delta N(\mathbf{r})+e N \operatorname{div} \mathbf{u}(\mathbf{r})=0 .
$$

It follows from the equations $(1),(2),(6)$ that:

$$
\operatorname{curl}\{(1-4 \pi \chi) \mathbf{b}(\mathbf{r})\}=-4 \pi \operatorname{curl}\left\{\frac{\partial \mathbf{M}}{\partial \zeta} \frac{N}{N_{\zeta}^{*}} \operatorname{div} \mathbf{u}(\mathbf{r})\right\} .
$$

The set of simultaneous equations (1),(2),(6) was first presented in previous works $[1,6]$. We use these equations to exclude $\mathbf{b}(\mathbf{r})$ and to express the potential $\varphi(\mathbf{r})$ in terms of the lattice displacement vector. As a result we arrive at the expression for the electron force $\mathbf{F}(\mathbf{r})$ acting upon the lattice under its displacement by the vector $\mathbf{u}(\mathbf{r})$ :

$$
\mathbf{F}(\mathbf{r})=\lambda_{0} \mathbf{b}_{0}\left(\mathbf{b}_{0} \nabla(\nabla \mathbf{u}(\mathbf{r}))\right)+\lambda\left[\mathbf{b}_{0} \times\left[\nabla(\nabla \mathbf{u}(\mathbf{r})) \times \mathbf{b}_{0}\right]\right] .
$$

Here, $\mathbf{b}_{0}$ is the unit vector directed along $\mathbf{B}$. This result (8) proves that the constants $\lambda_{0}, \lambda_{1}$ represent electron contributions to the elastic constants corresponding to the deformation of the lattice along the external magnetic field $\left(\lambda_{0}\right)$ and across this field $(\lambda)$. Within the adopted geometry these constants equal the electron terms in the compression elastic moduli $c_{33}$ and $c_{11}=c_{22}$ (in Voight notation). On the basis of the equations (1), (2),(6) the expressions for these constants are derived $[1,6]$ :

$$
\begin{aligned}
\lambda_{0} & =\frac{N^{2}}{N_{\zeta}^{*}} \\
\lambda & =\lambda_{0}\left(1+\frac{4 \pi \chi_{\zeta}}{1-4 \pi \chi_{\|}}\right) .
\end{aligned}
$$

Here $\chi_{\|}=\frac{\partial M_{z}}{\partial B}+\frac{\partial M_{z}}{\partial \zeta} \frac{\partial \zeta}{\partial B}$ is the longitudinal part of the magnetic susceptibility; $\chi_{\zeta}=\frac{\partial M_{z}}{\partial \zeta} \frac{\partial \zeta}{\partial B}$.

As follows from Eq.(9), the quantity $\lambda_{0}$ coincides with the compression modulus of the electron liquid. The structure of the quantity $\lambda$ is more complicated. Besides the electron compression contribution, $\lambda$ also contains a contribution of a different origin. This extra term appears due to the inhomogeneous magnetic field $\mathbf{b}(\mathbf{r})$ caused with the lattice deformation. This field arises due to the change in the magnetization of electrons caused by the deformation. So, the appearance of the second term in the expression (10) is a manifestation of a magnetostriction effect.

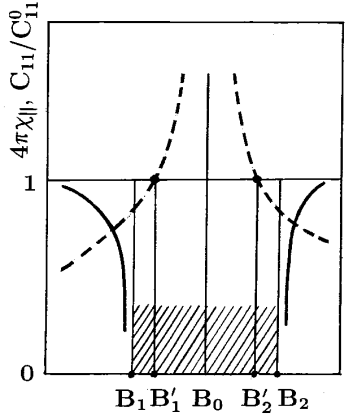

FIG. 1: Schematic plot of the magnetic field dependence of $4 \pi \chi_{\|}$(dashed line) and $c_{11} / c_{11}^{0}$ (solid line) near a peak of quantum oscillations at $B=B_{0}(T=0)$. The range of magnetic fields corresponding to structural $\left(B_{1}<B<B_{2}\right)$ and/or magnetic $\left(B_{1}^{\prime}<B<B_{2}^{\prime}\right)$ instability is section-lined.

When the differential magnetic susceptibility enhances in the proximity of diamagnetic phase transition, the denominator of the second term in (10) can take on values close to zero. Owing to this, the quantity $\lambda$ significantly grows in magnitude. For negative $\lambda$ this enhancement brings a noticeable decrease in the elastic constant $c_{11}$ (the latter is the sum of the "bare" elastic modulus $c_{11}^{0}$ and the electron contribution $\lambda$ ). In other words, the expression (10) reveals a possibility for softening of a longitudinal acoustic mode propagating perpendicularly to the magnetic field $\mathbf{B}$. This possible softening arises due to magnetostriction, and it results from magnetic instability.

When a strong magnetic field is applied to a metal, this gives rise to quantum oscillations in the electron DOS. The latter causes quantum oscillations in observables including magnetic susceptibility $\chi_{\|}$. At low temperatures $(\theta<<1)$ the magnitude of quantum oscillations increases so much that the oscillating term could predominate at peaks of oscillations. It was shown before (within the simple model of isotropic electron liquid) that under such conditions both $N_{\zeta}^{*}$ and $1-4 \pi \chi_{\|}$could go to zero near the oscillations peaks producing magnetic instability of the metal and softening of some acoustic modes $[1,5,6]$. This is illustrated in the Fig. 1. Here, magnetic fields $B_{1}^{\prime}$ and $B_{2}^{\prime}$ label thresholds of the magnetic instability region, and the differential magnetic susceptibility diverges at these points. Singularity in the longitudinal susceptibility $\chi_{\|}$discussed in the earlier papers [1], appears significantly closer to the field $B_{0}$ indicating the position of the oscillation peak. At the same time, the structural instability thresholds $B_{1}$ and $B_{2}$ are located farther from $B_{0}$ than $B_{1}^{\prime}$ and $B_{2}^{\prime}$, respectively, as shown in the Figure 1. However, these effects could be revealed in experiments only at extremely low temperatures (of the order of $10 \mathrm{mK}$ or lower). The stringent requirements for temperatures explain why the softening of the phonon modes 
at peaks of quantum oscillations was not observed so far.

It is known that under certain conditions local geometrical features of the FS could significantly affect the electronic response of the metal. This happens when a dominating contribution to the response functions results from small "effective" segments of the FS, where "efficient" electrons are concentrated. When the effective segments of the FS are nearly cylindrical or include locally flattened pieces, this gives a significant enhancement in the number of effective electrons, and can produce noticeable changes in the electronic response of the metal. The influence of locally flattened and neary cylindrical parts of the FS on the ultrasound attenuation rate, as well as on the surface impedance of a metall has been analyzed before (see e.g. [8-10]). Quantum oscillations of the electron DOS in strong magnetic fields are specified with contributions from effective cross-sections of the FS. Those are cross-sections with minimum and maximum sectional areas. Therefore the local geometry of the FS in the vicinities of these cross-sections has to affect both magnitude and shape of the oscillations [10]. This can influence anomalies of the elastic moduli under the present study and create much more favorable conditions for their observations in metals, as we show below.

We consider a metal whose FS is axially symmetric in the vicinity of an extremal cross-section at $p_{z}=p_{0}$ with the area $A_{e x}$. We assume the magnetic field $\mathbf{B}$ to go along the symmetry axis. The general expression for the FS curvature near $p_{z}=p_{0}$ could be written in the form:

$$
K(x)=-\frac{1}{2} \frac{d^{2} A / d x^{2}}{p_{m}^{2} A_{e x}} .
$$

Here, $x=\left(p_{z}-p_{0}\right) / p_{m} ; p_{m}$ is the maximum value of the quasimomentum component $p_{z}$ at the FS. Now, we adopt the following approximation for the cross-sectional area around the extremal cross-section:

$$
A(x)=A_{e x}\left(1 \pm b^{2} x^{2 l}\right)
$$

where $b^{2}$ is the dimensionless constant, $\left(b^{2}<<1\right)$ and the parameter $l$ takes on values greater than 1 .

The very essence of the employed model (12) is that it describes an axially symmetric FS whose curvature turns zero at $p_{z}=p_{0}$. The expression for the curvature (11) showes that the approximation (12) is necessarily applicable to each nearly cylindrical strip on any such FS. Otherwise the strip has a nonzero curvature. So we see that the model (12) gives the general expression for cross-sectional area of any nearly cylindrical segment of an axially symmetric FS.

Assuminig that the cyclotron quantum $\hbar \Omega$ is small compared to $\zeta\left(\gamma^{-1} \equiv(\hbar \Omega / \zeta)^{1 / 2}<<1\right)$ and using the model (12) we arrive at the following expression for the contribution from the nearly-cylindrical cross-section to the electron DOS oscillations:

$$
\Delta=\frac{\eta}{(\gamma)^{1 / l}} \sum_{r=1}^{\infty} \frac{(-1)^{r}}{r^{1 / 2 l}} \psi_{r}(\theta) \cos \left(\pi r \gamma^{2} \pm \frac{\pi}{4 l}\right) \cos \left(\pi r \frac{\Omega_{0}}{\Omega}\right)
$$

Here, $\quad \psi_{r}(\theta)=\frac{r \theta}{\sinh r \theta} ; \eta=\frac{\Gamma(1 / 2 l)}{2 l(b \sqrt{\pi})^{1 / l}} ; \Gamma(x) \quad$ is the gamma function, and $\hbar \Omega_{0}$ is the spin splitting energy. Our formula (13) agrees with the results obtained for a precisely cylindrical FS/and a Fermi circle in a twodimensional conductor (see e.g. [11,12]). We arrive at the corresponding results within the limit $l \rightarrow \infty$. In general case we can treat " $l$ " as a phenomenological parameter included in the model (12). Actual values of " $l$ " could be discovered in experiments where the FS local geometry is revealed. This is the only trustworthy way to estimate the above parameter for a particular metal. First principle calculations are not accurate enough to produce reliable results on fine geometrical features of FSs although their shapes as a whole are well known.

Within the isotropic model the cross sectional area is described with the expression (12) where $l=b^{2}=$ $1, p_{0}=0$, and $p_{m}$ is the radius of the Fermi sphere in quasimomemta space. In this particular case the oscillating function $\Delta$ takes on a well-known form:

$$
\Delta=\frac{1}{\gamma} \sum_{r=1}^{\infty} \frac{(-1)^{r}}{\sqrt{r}} \psi_{r}(\theta) \cos \left(\pi r \gamma^{2}-\frac{\pi}{4}\right) \cos \left(\pi r \frac{\Omega_{0}}{\Omega}\right) .
$$

Oscillations described by (13) and (14) differ in phase as well as amplitude. The amplitude of usual oscillations given by (14) is of the order of $\gamma^{-1} \theta^{-1 / 2}$, while (13) gives a magnitude of the order of $\gamma^{-1 / l} \theta^{(1-2 l) / 2 l}$. Therefore, the amplitude of oscillations related to the extremal section of zero curvature is approximately $\left(\gamma^{-1} \theta^{1 / 2}\right)^{(1-l) / l}$ times greater than that of the usual quantum oscillations. As a result, the contribution due to an extremal section with zero curvature can be considerably (more that tenfold) greater than contributions due to other extremal sections, and the function $\Delta$ can reach values of the order of unity at peaks of oscillations even at $\theta \sim 1$. On these grounds we conclude that the most favorable conditions for observation of softening of elastic moduli at peaks of quantum oscillations occur in metals whose FSs include nearly cylindrical segments. We consider such FSs in further analysis.

Assuming $\gamma<<1$ we replace matrix elements included in the Fermi-liquid kernel with their semiclassical analogs $\varphi\left(\mathbf{p}, \mathbf{p}^{\prime}\right)$ and $\psi\left(\mathbf{p}, \mathbf{p}^{\prime}\right)$ which depend on quasimomenta of interacting conduction electrons $\mathbf{p}$ and $\mathbf{p}^{\prime}$. For axially symmetric FSs the Fermi-liquid functions take the form:

$$
\begin{aligned}
& \varphi\left(\mathbf{p}, \mathbf{p}^{\prime}\right)=\varphi_{00}+p_{z} p_{z}^{\prime} \varphi_{01}+\left(\mathbf{p}_{\perp} \mathbf{p}_{\perp}^{\prime}\right)\left(\varphi_{10}+p_{z} p_{z}^{\prime} \varphi_{11}\right) ; \\
& \psi\left(\mathbf{p}, \mathbf{p}^{\prime}\right)=\psi_{00}+p_{z} p_{z}^{\prime} \psi_{01}+\left(\mathbf{p}_{\perp} \mathbf{p}_{\perp}^{\prime}\right)\left(\psi_{10}+p_{z} p_{z}^{\prime} \psi_{11}\right) .
\end{aligned}
$$


To make our analysis more thorough we include deformation terms in the force (8) exerted by the conduction electrons on the lattice. We present the components of the deformation potential, as:

$$
\Lambda_{\alpha \beta}(\mathbf{p})=\Lambda_{\alpha \beta}+G \Pi_{\alpha \beta}(\mathbf{p}),
$$

where $\Lambda_{\alpha \beta}$ is a tensor whose elements do not depend on $\mathbf{p}, G$ is a dimensionless constant, and $\Pi_{\alpha \beta}$ is the tensor of the electron momentum flux density.

The adopted approximations lead to the following expressions for the corrections to the elastic constants:

$$
\begin{gathered}
\tilde{c}_{11}=\tilde{c}_{22}=-\frac{N^{2}}{g} \frac{\left[1+G /\left(1+A_{2}\right)\right]^{2} \Delta}{1+\left(1+W-4 \pi \chi_{0} \gamma^{4}\right) \Delta} \\
\tilde{c}_{12}=-\frac{N^{2}}{g}\left(\frac{G}{1+A_{2}}\right)^{2} \frac{\Delta}{1+\left(1+W-4 \pi \chi_{0} \gamma^{4}\right) \Delta} .
\end{gathered}
$$

where $g$ is the electron DOS in the absence of the external magnetic field,

$$
\tilde{c}_{33}=-\frac{N^{2}}{g} \frac{\left[1+G\left(1+A_{2}\right)^{2}\right] \Delta}{1+(1+W) \Delta}
$$

$\chi_{0}$ is related to the Landau diamagnetic susceptibility (the latter equals $-\frac{1}{3} \chi_{0}$ ); and the constant $W$ originates from the Fermi-liquid interaction:

$$
W=\frac{B_{0}}{1+B_{0}}+\frac{B_{2}}{1+B_{2}}+\frac{A_{2}}{1+A_{2}} .
$$

Here, dimensionless coefficients $B_{0}, B_{2}$ and $A_{2}$ are related to the Fermi-liquid parameters $\psi_{00}, \psi_{11}$, and $\varphi_{11}$ as follows:

$$
\begin{aligned}
& B_{0}=\frac{1}{(2 \pi h)^{3}} \int \psi_{00} S\left(p_{z}\right) d p_{z} \\
& B_{2}=\frac{1}{(2 \pi h)^{3}} \int \psi_{11} S\left(p_{z}\right) p_{z} d p_{z} \\
& A_{2}=\frac{1}{(2 \pi h)^{3}} \int \varphi_{11} S\left(p_{z}\right) p_{z} d p_{z}
\end{aligned}
$$

It is noteworthy to mention that the shear modulus $c_{12}$ is also affected due to magnetostriction. This was not discovered in the early analysis of [1] where the deformation contributions into the electron force were omitted. As for elastic constant $c_{33}$, it also can be reduced at the peaks of low temperature quantum oscillations but this effect is not related to magnetic instability. The expression for $\tilde{c}_{33}(20)$ does not include the contribution arising due to magnetostriction. The possible softening of $c_{33}$ is directly connected with the behavior of the electron DOS under strong magnetic fields at low temperatures. Such instability was predicted before [7]. We have to remark here that the interaction among electrons significantly influences all above effects. The value of the constant
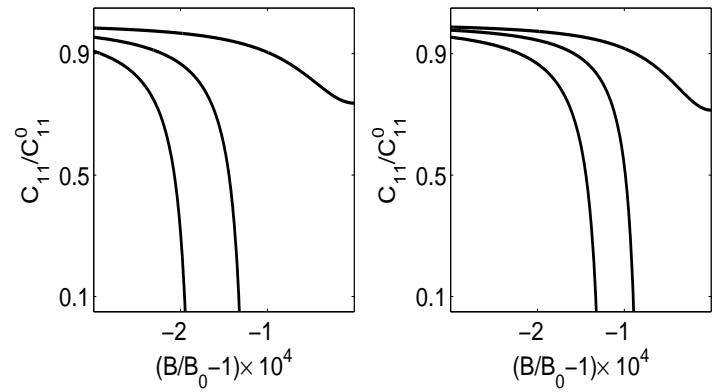

FIG. 2: Magnetic feld dependencies of the elastic constant $C_{11}$ near the diamagnetic phase transitions. The curves plotted for: $\theta=1$, the parameter $l$ takes on values $8,4,2$ from the left to the right (left panel); $l=4, \theta$ takes on values $1,2,3$ from the left to the right (right panel). For all curves $N=10^{21} \mathrm{~cm}^{-3}, \gamma^{2}=10^{3}, B_{0}=10 T$.

$W$ which accumulates effects of electron-electron interactions within the framework of the Fermi liquid theory, could significantly influence the temperature range where both magnetic and lattice instabilities occur.

The function $\Delta$ describing quantum oscillations is given by Eq. (13) and the amplitude of oscillations may become comparable with unity at moderately low temperatures provided that the FS shape reveals a fair proximity to a cylinder near the extremal cross section. For example, at $l=3, h \Omega / \zeta \sim 10^{-3}$, and $B \sim 10 T$, the condition $\gamma^{-1 / l} \theta^{(1-2 l) / 2 l} \sim 1$ could be satisfied at temperatures of the order of $1 \mathrm{~K}$. In usual metals $N \sim 10^{21} \div 10^{22} \mathrm{~cm}^{-3}$, and the term $4 \pi \chi_{0} \gamma^{4}$ in the denominator of (18) can take on values of the order of 10 at $\gamma^{2} \sim 10^{3}$. Also, we introduce the quantity $L^{2}=N^{2}\left(1+G /\left(1+A_{2}\right)\right)^{2} / g c^{0}$, where $c^{0}$ is the relevant "bare" elastic constant. The ratio $N^{2} / g c^{\circ}$ in typical metals is rather small $\left(N^{2} / g c^{0} \sim 10^{-2} \div 10^{-1}\right)$. However, the values taken on by $L^{2}$ could be noticeably greater than that due to the deformation constant $G$. The latter mostly accepts values of the order of unity. So, we have grounds to expect the values of $L^{2}$ to be of the order of $10^{-1}$ rather than $10^{-2}$.

To proceed in the analysis of the experimental feasibility of the effect, we numerically evaluate the decrease in the elastic constant $c_{11}$ using our result (18) and the above estimations of the parameters included there. The results are shown in the Fig. 2. We see that close enough proximity in the shape of an effective strip on the FS to a cylinder gives rise to the structural instability near peaks of oscillations at $\theta=1$. Also, it is demonstrated that the effect is washed out due to the further rise in temperature.

Electronic contributions to the velocity of ultrasound waves propagating in metals are simply related to the elastic constants. It follows from our results that the velocity of a longitudinal sound could depend on the di- 
rection of its propagation. In the vicinity of the Condon instability the velocity of sound propagating perpendicularly to the magnetic field $\mathbf{B}$ could be noticeably reduced compared to the velocity of sound propagating along $\mathbf{B}$. Again, we may expect this effect to appear in metals whose FSs include nearly cylindrical segments.

Finally, it is known that the effect of magnetostriction can cause softening of some phonon modes in metals near the Condon magnetic instability. This effect can appear even in an isotropic metal $[1,6]$. The point of the present work is that the effects could be significantly strengthened when the immediate vicinities of some extremal cross-sections of the FS are nearly cylindrical in shape, so that the FS curvature turns zero at these crosssections [13].there is an Real metals mostly have nonspherical and complicated in shape FSs. At present the main geometric characteristics of the FSs, such as their connectivity, are there is an well studied. On the contrary, local geometric features of the FSs has not been investigated in detail so far. However, there is an experimental evidence that "necks" connecting quasispherical pieces of the FS of copper include nearly cylindrical belts [11]. When the magnetic field is directed along the axis of a "neck" (for instance, along the [111] direction in the quasimomenta space), the extremal cross section of the "neck" could be expected to run along the nearly cylindrical strip where the FS curvature turns zero. It is also likely that the FS of gold possesses the same geometrical features for it closely resembles that of copper. Another kind of materials where we can expect the low temperature softening of phonon modes to be manifested includes some layered structures with metallic-type conductivity (e.g. $\alpha-(B E D T-T T F)_{2} M H g(S C N)_{4}$ group of organic metals). Fermi surfaces of these materials are sets of rippled cylinders, isolated or connected by links [14]. Based on the experiments on cyclotron resonance in these organic metals [15], it was shown that the cylinders could have nearly cylindrical strips [16]. For all above listed substances we can expect the effect to be revealed at reasonably low temperatures $(\sim 1 K)$ and reasonably strong magnetic fields $(1 \div 10 T)$.

The effect is expected to be very sensitive to the geometry of an experiment for extremal cross sections of the FS run along nearly cylindrical belts (if any) only at certain directions of the magnetic field. When the magnetic field is tilted away from such direction, the extremal cross section slips from the nearly cylindrical piece of the FS. This does not cancel possibilities of the effect in principle but makes requirements for temperatures dramatically stringent, as it was discussed above. The present analysis is carried out within the model of axially symmetric FS. The obtained results could be applied to actual metals when the magnetic field is directed in parallel with a high order axis of symmetry of the crystalline lattice of a metal. Otherwise, it is very difficult to separate out electron contributions to particular elastic constants. In summary, the effect of softening of phonon modes at peaks of quantum oscillations in metals could be expected to be observed in experiments. The most favorable conditions for the effect exist in metals whose FSs include nearly cylindrical pieces. The efffect could be revealed in these metals for some particular directions of the external magnetic field providing that an extremal cross section belongs to a quasicylindrical strip on the FS.

Acknowledgments: I thank G.M.Zimbovsky for help with the manuscript. This work was supported in part by NSF Advance program SBE-0123654.

1. V.N. Bagaev, V.I. Okulov, and E.A. Pamyatnykh, Zh. Eksp. Teor. Fiz. Pis'ma 27, 156 (1978) [JETP Lett. 27, 144 (1978)].

2. Ya.M. Blanter, M.I. Kaganov, D.B. Posvyanskii, Usp. Fiz. Nauk 165, 213 (1995) [Phys.-Usp. 165, 178 (1995)].

3. Similar results for two-dimensional systems are presented in: G. Montambaux, M. Heriter and P. Lederer, Phys. Rev. Lett. 55, 2078 (1985).

4. For the most recent review on the theory of diamagnetic phase transitions in metals see: A. Gordon, I.D. Vagner and P. Wyder, Adv. Phys. 52, 385 (2003).

5. L.R. Testardi and J.H. Condon, Phys. Rev. B, 1, 3928 (1970)

6. N.A. Zimbovskaya, V.I. Okulov and E.A. Pamyatnykh, Fiz. Met. and Metalloved. 54, 224 (1982) [In Russian].

7. B.T. Lazarev, E.A. Kaner and L.V. Chebotarev, Fiz. Nizk. Temp. 4, 808 (1978) [Sov. J. Low Temp. Phys. 3, 394 (1977)]; E.A. Kaner, L.V. Chebotarev and E. Uvimana, Fiz. Nizk. Temp. 4, 1218 (1978) [Sov. Phys. J. Low Temp. Phys. 4, 789 (1978)].

8. V.M. Kontorovich, Usp. Fiz. Nauk 27, 216 (1984) [Sov. Phys.-Usp. 27, 134 (1984)].

9. M.I. Kaganov and Yu.V. Gribkova, Fiz. Nizk. Temp. 17, 907 (1991) [Sov. J. Low Themp. Phys. 17, 473 (1991)].

10. N.A. Zimbovskaya, Zh. Eksp. Teor. Fiz., 107, 1672 (1995) [JETP 80, 932 (1995)]; N.A. Zimbovskaya and J.L. Birman, J. Phys.: Condens. Matter 12, 3337 (2000).

11. D. Shoenberg, "Magnetic Oscillations in Metals", Cambridge University Press, New York, 1984; see also: N.A. Zimbovskaya, Local Geometry of the Fermi Surface and High-Frequency Phenomena in Metals. SpringerVerlag, NY, 2001.

12. D. Shoenberg, J. Low Temp. Phys. 56, 417 (1984).

13. It should be emphasized that the present results are mostly applied to convenient metals. One hardly could expect a cylindrical segment of a considerable size to be included in the FSs of such metals. The local geometrical features of the FSs are crucially important for the phonon modes softening to be revealed there.

14. J. Wosnitsa, "Fermi Surface of Low-Dimensional Organic Metals and Superconductors", Springer, Berlin, 1996.

15. S.V. Demishev, A.V. Semeno, N.E. Sluchanko, et al., Phys. Rev. B 53, 12794 (1996).

16. N.A. Zimbovskaya, Zh. Eksp. Teor. Fiz., 113, 1965 (1998) [JETP, 86, 1220 (1998)]. 